\title{
Genome-wide profiling of mouse RNA secondary structures reveals key features of the mammalian transcriptome
}

\author{
Danny Incarnato ${ }^{1,2}$, Francesco Neri ${ }^{1}$, Francesca Anselmi ${ }^{1}$ and Salvatore Oliviero ${ }^{1,3^{*}}$
}

\begin{abstract}
Background: The understanding of RNA structure is a key feature toward the comprehension of RNA functions and mechanisms of action. In particular, non-coding RNAs are thought to exert their functions by specific secondary structures, but an efficient annotation on a large scale of these structures is still missing.

Results: By using a novel high-throughput method, named chemical inference of RNA structures, CIRS-seq, that uses dimethyl sulfate, and N-cyclohexyl- N'-(2-morpholinoethyl)carbodiimide metho-p-toluenesulfonate to modify RNA residues in single-stranded conformation within native deproteinized RNA secondary structures, we investigate the structural features of mouse embryonic stem cell transcripts. Our analysis reveals an unexpected higher structuring of the $5^{\prime}$ and $3^{\prime}$ untranslated regions compared to the coding regions, a reduced structuring at the Kozak sequence and stop codon, and a three-nucleotide periodicity across the coding region of messenger RNAs. We also observe that ncRNAs exhibit a higher degree of structuring with respect to protein coding transcripts. Moreover, we find that the Lin28a binding protein binds selectively to RNA motifs with a strong preference toward a single stranded conformation.
\end{abstract}

Conclusions: This work defines for the first time the complete RNA structurome of mouse embryonic stem cells, revealing an extremely distinct RNA structural landscape. These results demonstrate that CIRS-seq constitutes an important tool for the identification of native deproteinized RNA structures.

\section{Background}

The development of high-throughput methods for the analysis of the epigenome and transcriptome have led to the discovery of thousands of previously unannotated transcripts $[1,2]$, many of which lack the ability to encode proteins [3-6], as further proven by genome-wide ribosome profiling approaches [7]. While mechanisms of action have been elucidated for a small fraction of these non-coding RNAs (ncRNAs), for most the ways by which they contribute to gene regulation still remain unclear. One of the most intriguing modes of action proposed for long ncRNAs (lncRNAs) is their potential to act as modular scaffolds for the assembly of large multi-protein complexes [4,8], although the mechanistic aspects of these interactions are largely unknown. As learned from small

\footnotetext{
* Correspondence: salvatore.oliviero@hugef-torino.org

'Human Genetics Foundation (HuGeF), via Nizza 52, Torino 10126, Italy ${ }^{3}$ Dipartimento di Scienze della Vita e Biologia dei Sistemi, Università di Torino, Via Accademia Albertina, Torino 13-10123, Italy

Full list of author information is available at the end of the article
}

nuclear ribonucleic particle (snRNP) complexes [9,10], most ncRNAs are thought to exert their functions by folding into locally stable secondary structures that may provide anchoring sites for interacting proteins. For example, it has been shown in Drosophila melanogaster that both MLE and MSL2 proteins of the MSL complex act by binding to conserved structural domains of the roX1/2 ncRNAs, which then mediate targeting to the X chromosome to regulate dosage compensation in fruitfly [11,12]. Furthermore, in differentiating mouse embryonic stem cells (ESCs), MLL1 protein has been shown to be required for the transcriptional activation of Hoxa6/7 genes, and its recruitment to chromatin is mediated by interaction with a stem-loop structure located in the 3 ' region of the Mistral lncRNA [13].

The growing number of annotated transcripts has outpaced the efficient analysis of their structure; at present, structural information exists for only a very tiny minority of annotated RNAs. To address this need, over the past few years various enzymatic- and chemical-based approaches 
have been proposed for the discovery of secondary structures for thousands of RNAs at a time [14-18]; however, all these methods are based on the assumption that in vitro folding may be representative of native RNA structures in vivo. While for certain small RNAs the in vitro folding landscape recapitulates well the in vivo one [19-21], long RNAs often exhibit rugged folding landscapes that lead in vitro to the prevalence of kinetically trapped intermediates and misfolded structures [22-24]. For example, in vitro folding of the RNAse $\mathrm{P}$ ribozyme is a slow process that takes several minutes and requires escape from a kinetic trap $[23,25]$. Comparative analysis of in vivo and in vitro probing data on human telomerase RNA revealed that while the 3'-terminal small nucleolar RNA (snoRNA)-like domain folds into comparable structures in the two conditions, the $5^{\prime}$ template domain exhibits very different foldings [26].

Two main scenarios can explain the differences observed in RNA folding in vitro and in vivo. The first is based on the assumption that, in the cell, most nascent transcripts are likely to fold during transcription $[20,27,28]$. In this perspective, the elongation rate of RNA polymerase, as well as the directionality of transcription, may influence the order and the speed of the folding events, thus preventing the formation of non-native, kinetically trapped intermediates [29]. The second, which does not exclude the first, is that many specific as well as non-specific RNA binding proteins (RBPs) may act as RNA chaperones, thus directing and stabilizing RNA folding [30-33]. To overcome the issues introduced by the study of RNA folding in vitro, two recent reports analyzed the structures of Saccharomyces cerevisiae and Arabidopsis thaliana RNAs by treating the cells with dimethyl sulfate (DMS) [34,35].

We present here a new method, named chemical inference of RNA structures followed by massive parallel sequencing (CIRS-seq), that allows genome-wide investigation of native deproteinized RNA secondary structures by exploiting the capacity of DMS and Ncyclohexyl-N'-(2-morpholinoethyl)carbodiimide metho-ptoluenesulfonate (CMCT) to specifically react with RNA unpaired bases. Our approach, applied to mouse ESCs, allowed us to obtain single-base resolution structural information for thousands of transcripts in their native deproteinized conformation, revealing the structural complexity of the mammalian transcriptome.

\section{Results}

\section{CIRS-seq enables accurate transcriptome-wide inference} of single-stranded RNA residues

The CIRS-seq method (Figure 1) is based on the use of DMS, which mainly methylates N1 of adenosine and N3 of cytosine [36,37], and CMCT, which primarily forms adducts with N1 and N3 of pseudouridine, N3 of uridine, and, to a lesser extent, N1 of guanosine and inosine [38-40] but only when these residues are in single-stranded conformation. Treatment of RNA with the two reagents enables the detection of unpaired nucleotide positions due to the modification-induced reverse transcription (RT) stop one nucleotide downstream of the modified residue. To carry out CIRS-seq, we first optimized treatments to achieve similar degrees of modification with the two reagents at different concentrations, as measured by reduction of the full-length reverse transcription product for a test RNA following reaction with either DMS or CMCT (Figure $\mathrm{S} 1$ in Additional file 1).

To perform transcriptome-wide probing of RNAs in their native deproteinized conformation, we lysed mouse ESCs in an isotonic buffer, and treated the lysate with Proteinase $\mathrm{K}$ to unmask regions of RNAs bound by proteins, without affecting the RNA structure (supplementary Materials and methods in Additional file 1). ESC lysates were then treated with DMS or CMCT, and total RNA was extracted following reaction quenching. Extracted RNA was subjected to random-primed RT. A non-treated control was also produced to determine naturally occurring RT stops. The generated cDNAs were adapter ligated and subjected to high-throughput sequencing using the Illumina platform, resulting in about $90 \times 10^{6}$ deep-sequencing reads for each treatment, across two biological replicates.

Since proper analysis of RNA folding requires correct annotation of transcript sequences, reads were mapped to a recently published variant of the $\mathrm{mm} 9$ assembly that integrates single-nucleotide variants from the E14 ESC line [41], and we obtained a similar distribution of read mappings across all samples (Figure S2A in Additional file 1). Estimation of transcript abundances using CIRSseq data correlated well across treatments, and with canonical RNA-seq data $(R \geq 0.9$, Spearman correlation; Figure S2B in Additional file 1), showing that the CIRSseq method enables unbiased probing of RNAs. At the current coverage, we obtained structural information for approximately 30,000 transcripts, belonging to approximately 13,000 genes (Figure 2a; Figure S2C in Additional file 1).

As a quantitative measure of the probability of observing a RT stop specifically induced by our treatment, we calculated raw reactivity scores as the base 2 logarithm ratio of the normalized read counts for the DMS/CMCT treatment at a given position of a transcript, and the normalized read counts at the same position in the nontreated control. The final normalization (Supplementary methods in Additional file 1) yielded reactivity values ranging from 0 to 1 , and positions with reactivities $>0$ and $<0.3,0.3$ to 0.7 , or $>0.7$ were designated as weakly, moderately, or highly reactive, respectively [42]. Correlation analysis of reactivity values across the top 75th 


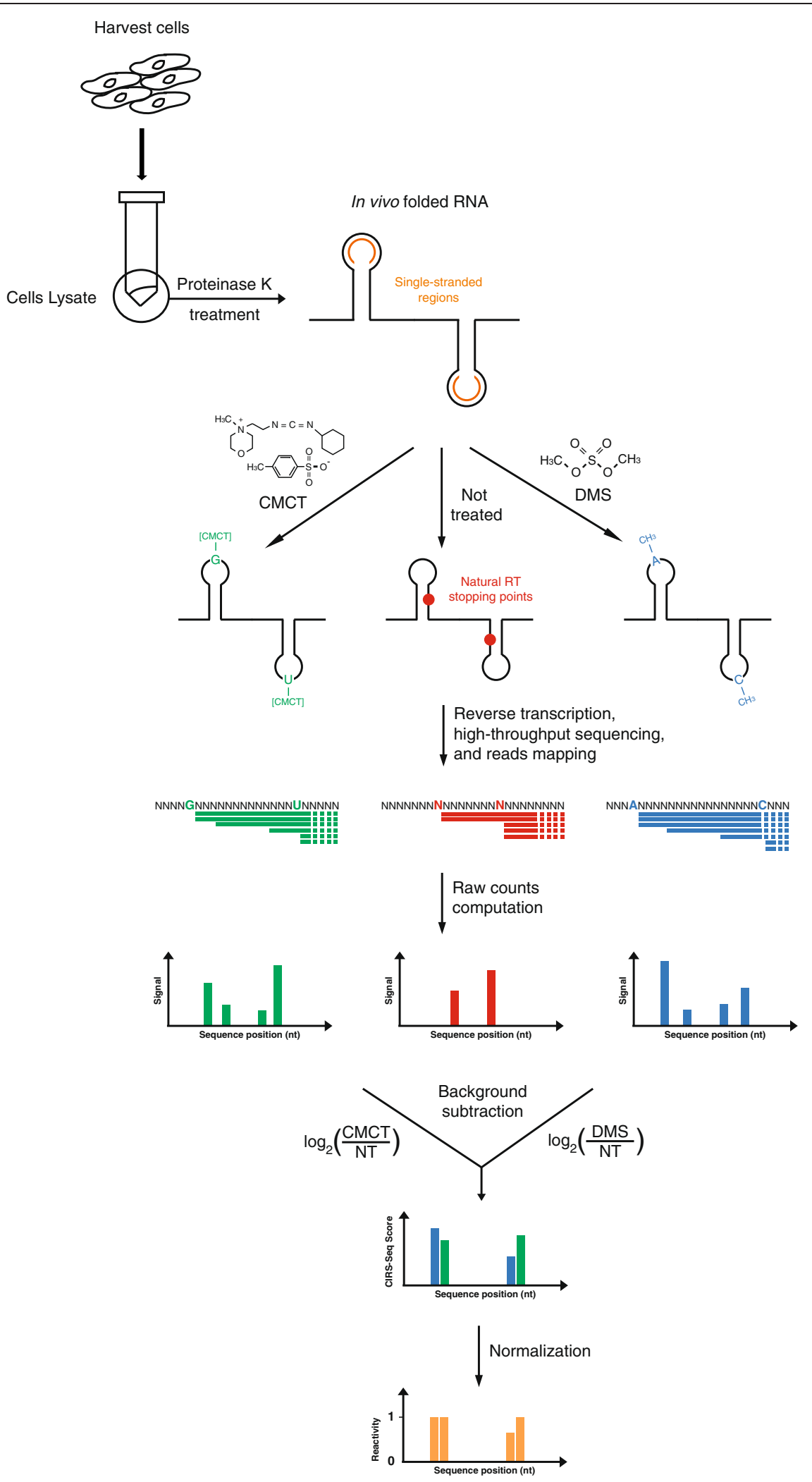

Figure 1 (See legend on next page.) 
(See figure on previous page.)

Figure 1 Overview of the CIRS-seq method. Cells are harvested and lysed in isotonic buffer, then treated with Proteinase $\mathrm{K}$ to unmask protein-bound regions of RNAs. The whole cell population of RNAs in their native deproteinized conformation is probed with either DMS or CMCT to modify unpaired bases. A non-treated control is also produced to allow further mapping of natural RT stops. After modification, the RNAs from the three populations are reverse transcribed, and cDNA is adapter ligated for high-throughput sequencing. Mapping reads to the transcriptome provide information regarding how many RT stops occurred at each position of the analyzed transcripts. The non-treated (NT) signal at each position is then subtracted from the DMS and CMCT signals to obtain the raw reactivity profile at base resolution. After scaling each data point above the 90th percentile to the 90 th percentile, reactivity at each position is divided by the 90 th percentile $(90 \%$ Winsorising) to obtain the normalized reactivity.

percentile of covered transcripts revealed the high reproducibility of CIRS-seq $(R=0.90$, Pearson correlation; Figure $2 \mathrm{~b}$ ); therefore, we combined the two replicates for further analysis.

Collectively, we obtained structural data for 1,190,948 and 1,080,859 nucleotides in the DMS (weak, 13.6\%; moderate, 49.9\%; high, 36.5\%) and CMCT (weak, 16.6\%; moderate, $54.7 \%$; high, $28.7 \%$ ) treatments, respectively (Figure S2D in Additional file 1). To validate CIRS-seq, we overlaid reactivity data on the well characterized structures of tRNAs [43,44] (Figure 2c; Figure S3A,B in Additional file 1), and observed that all the highly reactive residues were almost completely confined to the tRNAs' D and anticodon arm loops, suggesting a high overall accuracy for our method.

Despite the respective strong preference of DMS and CMCT for $\mathrm{A} / \mathrm{C}$ and $\mathrm{G} / \mathrm{U}$ residues, we also observed non-canonical reactivities in both treatments. Our data are in agreement with previous reports showing DMS reactivity with $\mathrm{G} / \mathrm{U}$ residues $[36,45-47]$ and $\mathrm{CMCT}$ reactivity with cytosines [48-50]. We observed a significant increase in the accuracy of the de novo prediction of structures when considering also these non-canonical reactivities as both the canonical and non-canonical reactivities lay within single-stranded regions (Figure S3C in Additional file 1). Moreover, overlaying reactivity data on the known structures of U5 and U1 small nuclear RNAs (snRNAs) and U3 snoRNA (Figure 2d; Figure S4A,B in Additional file 1) showed that the Proteinase K treatment enabled high-resolution determination of secondary structures at the level of protein-masked regions of RNAs without losing the proper folding. In fact, internal loop IL2/IL2' of U5, box B/C of U3 and loop II of $\mathrm{U} 1$ are bound in vivo by, respectively, a $116 \mathrm{kDa}$ protein (Snu114p yeast homolog) [51], a $15.5 \mathrm{~K}$ protein [52,53], and the U1A protein [10]; these regions showed very high reactivity to DMS/CMCT treatments and were almost completely resolved by CIRS-seq. Overall, for the set of analyzed structures (Table 1), $80.6 \%$ of the highly reactive residues were located within single-stranded regions. Of the $19.4 \%$ of the highly reactive residues located within regions of the known structures annotated as double-stranded, $84.2 \%$ were positioned at the end of helices or adjacent to bulges/loops. These regions were previously shown to be subjected to structural flexibility, so chemical reagents can easily modify these terminal residues $[34,54]$. When accounting for these additional accessible positions, the overall true positive rate of our method rose to $96.3 \%$.

Collectively, this analysis proves the high accuracy of CIRS-seq, and provides a nucleotide-resolution panorama of the mouse ESC RNA structurome.

\section{CIRS-seq data allow accurate secondary structure prediction}

Next, we verified the ability of CIRS-seq to infer de novo secondary structures. Constraints derived from chemical probing data may significantly improve the accuracy of RNA secondary structure prediction tools $[42,56]$. We chose the U2 and low-abundance U12 snRNAs, and the valine and threonine tRNAs, whose structures were previously experimentally defined $[57,58]$, or can be easily derived from phylogenetic analysis. We used the RNAStructure tool [59] to devise secondary structures by imposing constraints for unpaired positions. This tool can accept chemical probing data in the form of SHAPE data files, allowing more comprehensive modeling of the structure according to the CIRS-seq-derived data compared with hard constraints-based methods. For both the unconstrained minimum free energy (MFE) and the CIRS-seq constrained secondary structures, we calculated the positive predictive value (PPV) as the fraction of base-pairs present in the predicted structure that are also present in the validated structure, and the sensitivity as the fraction of base-pairs present in the validated structure that are also in the predicted structure (Table 2). Notably, CIRS-seq-derived structures for all the four transcripts analyzed showed higher similarity to the known structures (Figure 3a,b; Figure S5A,B in Additional file 1); on average, the CIRS-seqguided folding outperformed the MFE unconstrained predictions in terms of both PPV and sensitivity (PPV 0.53 and sensitivity 0.57 for unconstrained MFE structures; PPV 0.95 and sensitivity 0.95 for CIRS-seq constrained structures). This analysis demonstrates that the use of CIRS-seq data improves the accuracy of RNA secondary structure prediction tools, and that low-abundance transcripts can be successfully probed by CIRS-seq. 

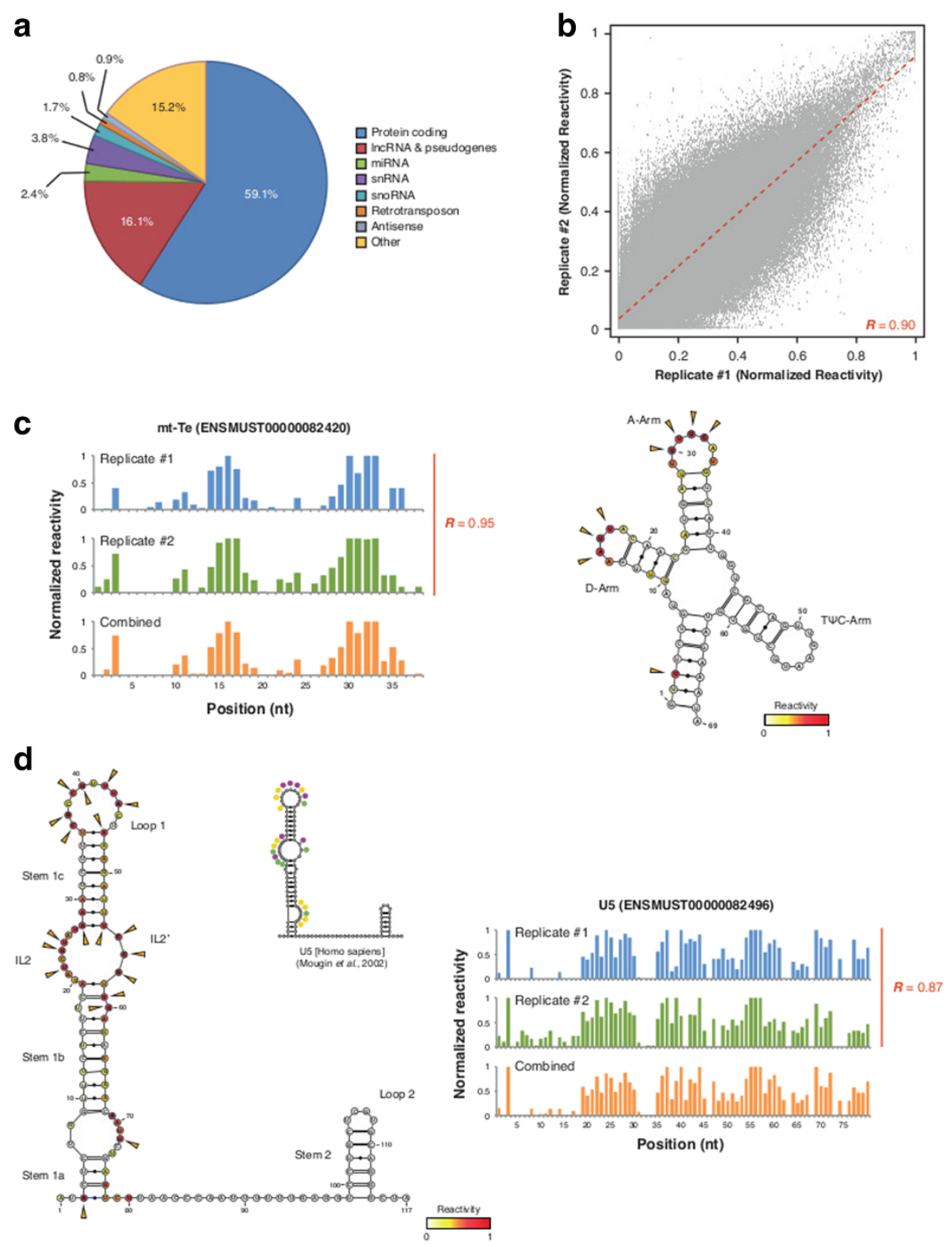

Figure 2 Validation of CIRS-seq data. (a) Distribution of transcripts with at least one RT stop on average per base. (b) Scatter plot of normalized reactivities in the two biological replicates of CIRS-seq. Reactivities are averaged in 10-nucleotide windows, with an offset of 5 nucleotides (Pearson's correlation coefficient $=0.90$. (c) Normalized reactivity profiles for the glutamic acid tRNA and overlay of reactivity data on the phylogenetically derived secondary structure. Yellow arrows indicate highly reactive positions (reactivity $>0.7$ ). Bases are color coded according to their reactivity. (c) Normalized reactivity profiles for the U5 snRNA and overlay of reactivity data on phylogenetically derived secondary structure. The structure of the U5 human homolog is also shown, with superimposed DMS/CMCT-reactive positions from [55]. The colors correspond to different degrees of chemical modification (purple, strong; yellow, medium; green, weak). Yellow arrows indicate highly reactive positions (reactivity $>0.7$ ). Bases are color coded according to their reactivity.

\section{CIRS-seq reveals structural features of mammalian mRNAs and ncRNAs}

Thanks to the high resolution enabled by CIRS-seq, we then investigated the structural features of mouse mRNAs, and looked for structural differences across transcript regions. We selected approximately 9,500 mRNAs, in which the DMS/CMCT treatment induced, on average, at least one RT stop per nucleotide (Supplementary methods in Additional file 1).

Meta-analysis of average reactivity across UTRs and coding regions revealed a strong reduction of reactivity scores in the 50 nucleotides of the 5' UTR immediately preceding the Kozak sequence (average 0.165) compared with the first and last 100 nucleotides of the coding 
Table 1 CIRS-seq efficiency on validated secondary structures

\begin{tabular}{|c|c|c|c|c|c|}
\hline \multirow[b]{2}{*}{ ENSEMBL ID } & \multirow[b]{2}{*}{ Symbol } & \multicolumn{2}{|c|}{ Including helix termini } & \multicolumn{2}{|c|}{ Excluding helix termini } \\
\hline & & TP (\%) & $\mathrm{FP}(\%)$ & TP (\%) & FP (\%) \\
\hline ENSMUST00000082420 & mt-Te & 87.5 & 12.5 & 87.5 & 12.5 \\
\hline ENSMUST00000082389 & mt-Ti & 75.0 & 25.0 & 100.0 & 0.0 \\
\hline ENSMUST00000082399 & $m t-T n$ & 90.0 & 10.0 & 90.0 & 10.0 \\
\hline ENSMUST00000083033 & U1 & 83.4 & 16.6 & 94.7 & 5.3 \\
\hline ENSMUST00000082496 & U5 & 73.7 & 26.3 & 100.0 & 0.0 \\
\hline ENSMUST00000082466 & U3 & 75.0 & 25.0 & 96.9 & 1.1 \\
\hline Total & & 80.6 & 19.4 & 96.3 & 3.7 \\
\hline
\end{tabular}

Percentages of true positive (TP) and false positive (FP) highly reactive positions for known secondary structures.

region (average $0.208, P$-value 3.0e-374, Wilcoxon rank sum test; Figure 4a,b). Moreover, a significant reduction of reactivity was also observed in the first 50 nucleotides of the 3' UTR immediately downstream of the stop codon (average 0.172, P-value 1.1e-243, Wilcoxon rank sum test). These results differ from what has been recently observed in $A$. thaliana, where the coding region is more structured than the UTRs [34]. We also identified a significant increase of reactivity score at the level of the Kozak sequence (average 0.229) with a maximum of reactivity on the base immediately preceding the AUG (average 0.345), and on the stop codon beginning three nucleotides upstream (average 0.226), compared with the coding region ( $P$-values $4.0 \mathrm{e}-24$ and $6.5 \mathrm{e}-8$, respectively, Wilcoxon rank sum test; Figure $4 \mathrm{c}$ ), revealing a markedly reduced probability of base-pairing in these regions. The reduced base-pairing on the Kozak sequence and around the stop codon suggests that a more accessible context in these regions of protein-coding transcripts may facilitate both the entry and the detachment of ribosomes.

We next analyzed the first and last 99 nucleotides of mRNA coding regions to determine if the previously reported periodic signal of three nucleotides [14,34] was conserved also in mouse. To this end, we observed that mouse protein-coding transcripts, similar to A. thaliana and $S$. cerevisiae mRNAs, exhibit a strong threenucleotide periodicity across the coding region that was not observed within the UTRs (Figure 4d). The second and third nucleotides of each codon were highly structured and exhibited lower average reactivities (average 0.205 and 0.199 , respectively), with the third nucleotide being the less reactive ( $P$-value $1.7 \mathrm{e}-07$, Wilcoxon rank sum test), while the first nucleotide was the less structured and significantly more reactive to DMS/CMCT treatment than the second and third (average 0.220, Pvalues $2.0 \mathrm{e}-12$ and $8.2 \mathrm{e}-12$, respectively, Wilcoxon rank sum test). Taken together these results suggest a deep involvement of RNA secondary structures in driving and regulating translation efficiency.

Analysis of the RNA structure is particularly relevant for ncRNAs as they are thought to exert their function by interacting with other molecules via their secondary structure. We then sought to determine whether an overall structural difference exists between protein coding RNAs and different classes of ncRNA transcripts. To avoid biases due to differential coverage, only transcript positions with sequencing depth greater than $50 \times$ were considered (Supplementary methods in Additional file 1). Analysis of normalized reactivity showed a significantly lower average reactivity of snoRNAs (average $0.282, P$-value $1.1 \mathrm{e}-87$, Wilcoxon rank sum test), snRNAs (average 0.295 , $P$-value $2.1 \mathrm{e}$ 146, Wilcoxon rank sum test), tRNAs (average 0.251, $P$-value 2.5e-9, Wilcoxon rank sum test), and long intergenic non-coding RNA (lincRNAs; average 0.309, $P$-value 7.4e-48, Wilcoxon rank sum test) compared with mRNAs (average 0.366) (Figure 4e). Collectively, these data reveal a higher structuring of ncRNA transcripts compared with mRNAs.

Table 2 Statistics for CIRS-seq de novo inferred secondary structures

\begin{tabular}{|c|c|c|c|c|c|}
\hline \multirow[b]{2}{*}{ ENSEMBL ID } & \multirow[b]{2}{*}{ Symbol } & \multicolumn{2}{|c|}{ Unconstrained (MFE) } & \multicolumn{2}{|c|}{ CIRS-seq constrained } \\
\hline & & PPV & Sensitivity & PPV & Sensitivity \\
\hline ENSMUST00000101806 & U2 & 0.68 & 0.89 & 1.00 & 1.00 \\
\hline ENSMUST00000083242 & U12 & 0.80 & 0.84 & 1.00 & 0.95 \\
\hline ENSMUST00000082389 & $m t-T v$ & 0.22 & 0.20 & 1.00 & 1.00 \\
\hline ENSMUST00000083422 & $m t-T t$ & 0.41 & 0.35 & 0.81 & 0.85 \\
\hline Average & & 0.53 & 0.57 & 0.95 & 0.95 \\
\hline
\end{tabular}

Positive predictive value (PPV) and sensitivity measures calculated for both the unconstrained minimum free energy (MFE) and CIRS-seq constrained structures. 


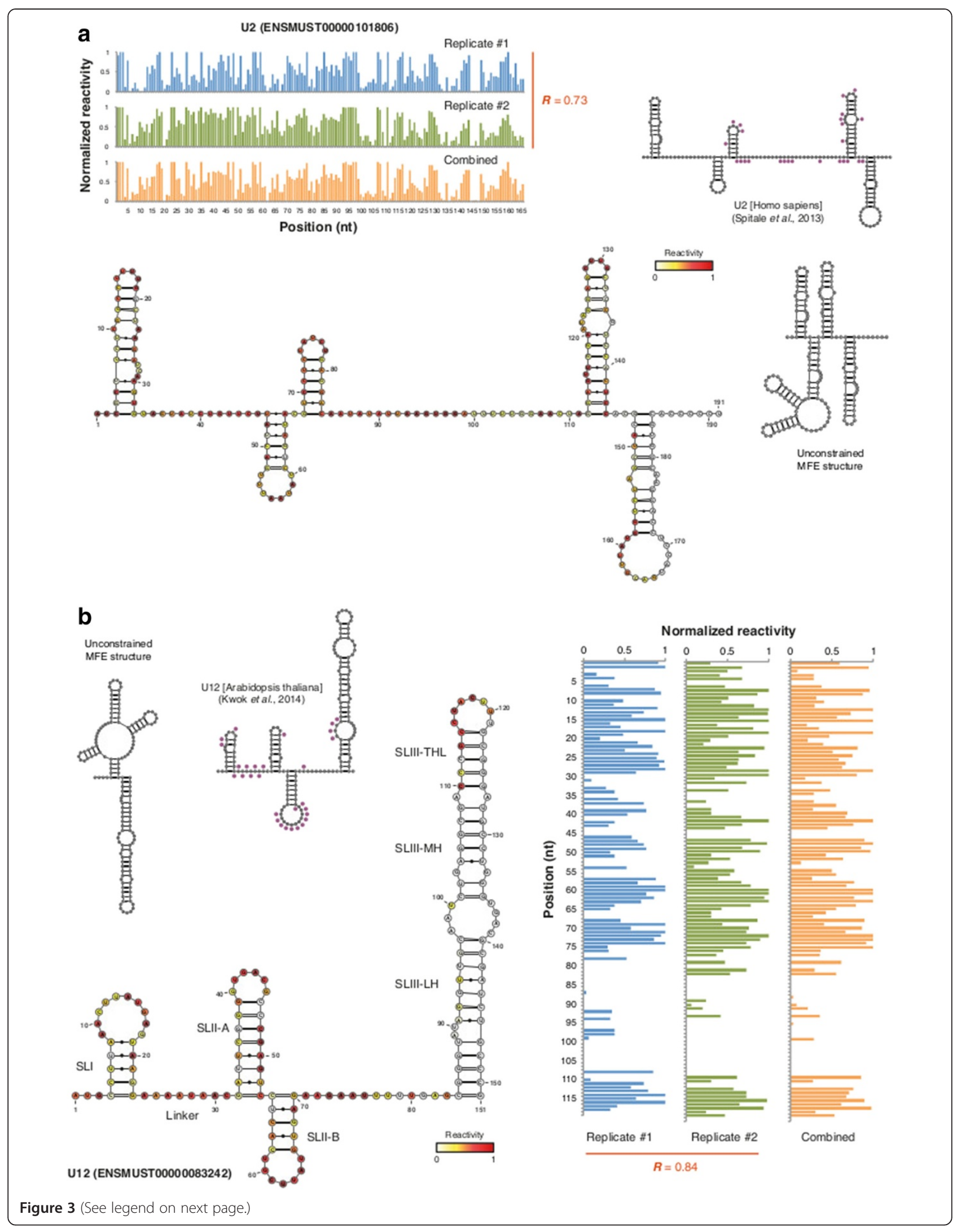


(See figure on previous page.)

Figure 3 CIRS-seq data allow correct inference of native deproteinized RNA secondary structures. (a) Normalized reactivity profiles for the U2 snRNA and overlay of reactivity data on the secondary structure inferred from chemical constraints. Bases are color coded according to their reactivity. The structure of the human ortholog with superimposed SHAPE-reactive positions from [57], and the unconstrained MFE structure are also shown. (b) Normalized reactivity profiles for the low-abundance U12 snRNA and overlay of reactivity data on the secondary structure inferred from chemical constraints. Bases are color coded according to their reactivity. The structure of the U12 A. thaliana ortholog with superimposed DMS/SHAPE-reactive positions from [58], and the unconstrained MFE structure are also shown.

\section{CIRS-seq identifies structural requirements of RNA binding proteins}

RNA-protein interactions are strongly influenced by secondary structures. Determining the structural requirements for RBPs to bind to their cognate targets is required to understand their roles and mechanisms of action. To this end, we analyzed from a structural perspective the binding sites of the highly conserved RBP Lin28a. Lin28a is highly expressed in ESCs, and is one of the factors required for the reprogramming of human fibroblasts to induced pluripotent stem cells [60]. To investigate the structural requirements of Lin28a binding, we analyzed a previously published CLIP-seq dataset of Lin28a in ESCs [61]. We identified peaks of Lin28a enrichment across the mouse transcriptome, and calculated average reactivity on a window of 300 nucleotides surrounding summits of the peaks (Figure 5a). While more distal regions around the Lin28a peaks showed a level of reactivity comparable to that of the coding sequence (average 0.21), in agreement with a preferential positioning of Lin28a binding sites within this region, we observed a significant and progressive increase in the accessibility proceeding toward the peak summits (maximum $0.34, \pm 25$ nucleotides average $0.27, P$-value $6.2 \mathrm{e}$ 79 , Wilcoxon rank sum test). Concordant with this observation, analysis of putative Lin28a binding sites revealed that the target A/G-rich motifs tends to assume a single-stranded conformation within the loop regions of hairpin-like structures (Figure 5b). This result is in agreement with previous in silico predictions based on the analysis of Watson-Crick pair co-occurrence around the Lin28a consensus [61].

\section{Discussion}

In this work we have defined, for the first time, the complete RNA structurome of mouse ESCs. Our analysis revealed the structural features of mRNAs and ncRNAs, and identified the structural requirements for Lin28a RNA binding protein.

The introduction of the CIRS-seq method, which does not rely on a denaturation and re-folding approach, allowed us to massively probe RNAs in their natural context. By applying CIRS-seq to mouse ESC RNAs, we were able to probe protein-coding RNAs as well as ncRNAs in their native deproteinized conformation. Analysis of previously validated secondary structures showed that CIRS-seq is extremely precise, and RNA secondary structures inferred using CIRS-seq data to constrain folding algorithms exhibit higher accuracy than MFE structures predicted in the absence of chemical probing data. Moreover, the use of two compounds that modify distinct bases, together with the introduction of a deproteinization step, which enabled us to investigate protein-masked regions of transcripts without losing their correct folding, increased the resolution of our method.

The analysis of CIRS-seq data revealed a strong structural partitioning of protein-coding transcripts, revealing a higher degree of structuring of UTRs compared with coding regions. This was unexpected since it has been recently reported that in $A$. thaliana UTRs have a higher propensity to single-strandedness than coding regions [34]. This difference may represent evolutionary structural diversity between metazoans and plant RNAs, as suggested by previous in vitro [17] and in silico [62] analyses, or it could be explained by reduced accessibility of transcript coding regions to DMS treatment, due to the ribosome occupancy, in the absence of a deproteinization step. However, the agreement of our data with a recent nuclease-based analysis conducted in human lymphoblastoid cells [63] suggests that this structuring is conserved in mammalian mRNAs and may have a functional role.

The slightly higher reactivity observed for 3' UTRs compared with $5^{\prime}$ UTRs in mouse mRNAs may be representative of the preference of microRNA recognition elements, which are highly enriched in 3' UTRs [64], to reside within more accessible contexts $[65,66]$. It must be also noticed that structural regulatory elements in the 3' UTR are often short and dispersed in the UTR, which in many cases may be very long, thus leading to a lower overall structuring of this region compared with the $5^{\prime}$ UTR [67].

Our analysis of Lin28a protein recognition elements demonstrated genome-wide that binding sites for this protein tend to preferentially assume a single-stranded conformation. We moreover observed that Lin28a motifs tend to reside within loop regions of hairpin-like structures.

Furthermore, the analysis of ncRNAs revealed a higher overall degree of structuring compared with proteincoding transcripts, and showed that lincRNAs exhibit structural features intermediate to those of mRNAs and 

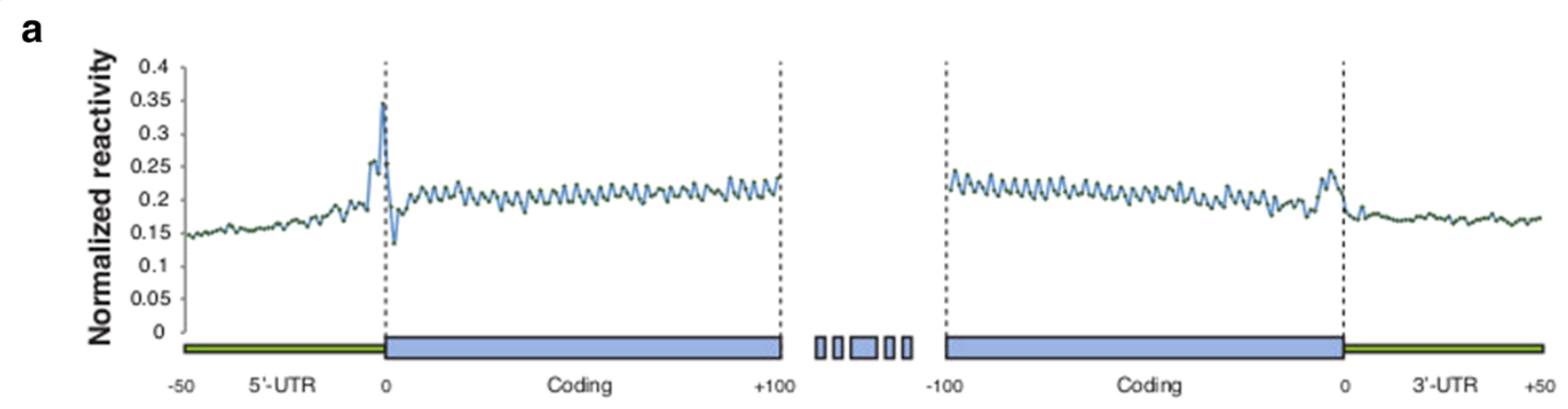

b
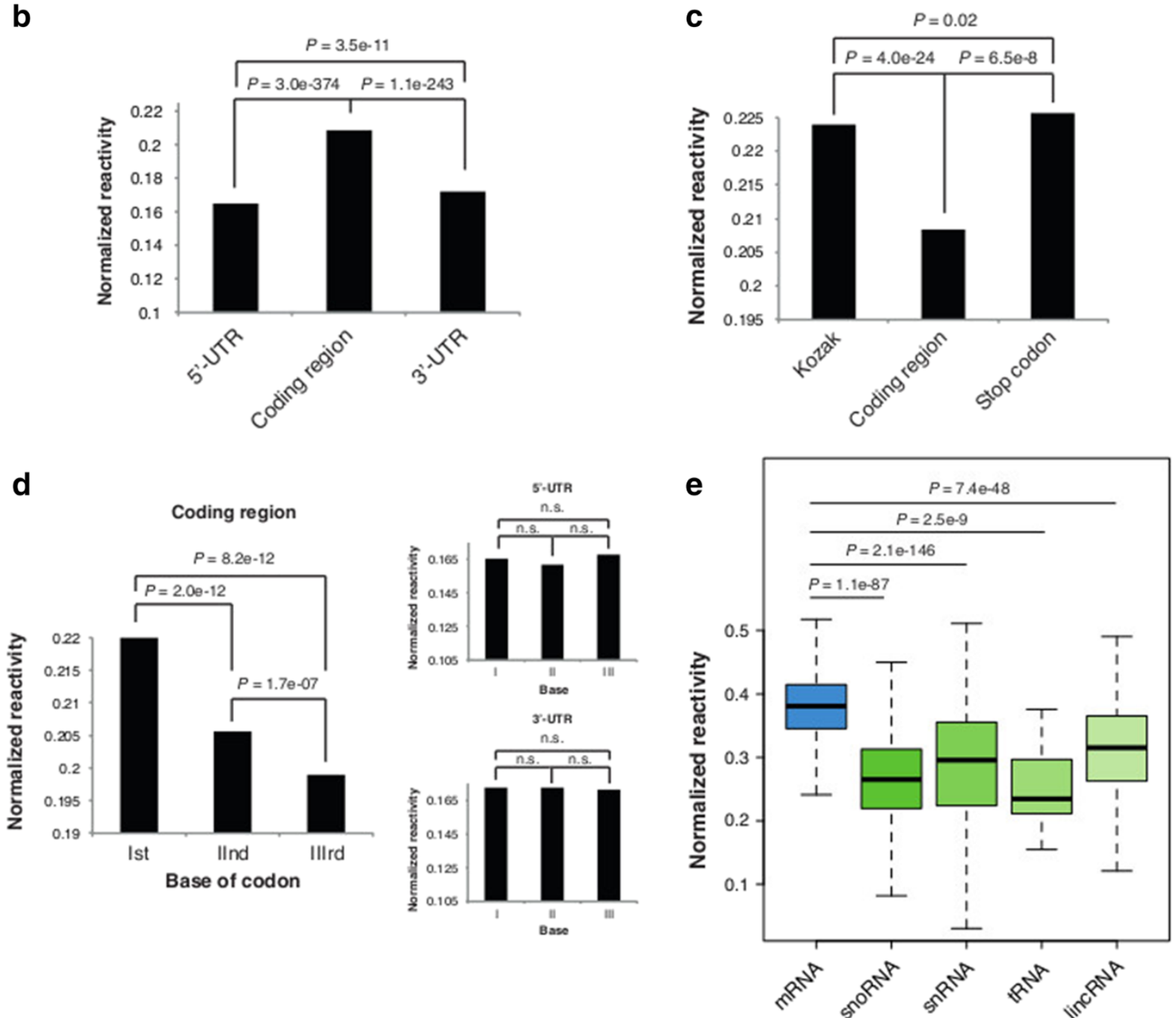

Figure 4 Transcriptome-wide analysis of mRNAs reveals structural features of protein-coding and non-coding transcripts. (a) Meta-gene analysis across the last 50 nucleotides of the $5^{\prime}$ UTR, the first and last 100 nucleotides of the coding region, and the first 50 nucleotides of the $3^{\prime}$ UTR of approximately 9,500 mRNAs. (b) Average reactivity of the 5' UTR, coding region, and 3' UTR. (c) Average reactivity on the Kozak sequence (-6/+1 nucleotides around AUG), coding region, and stop codon (+3 nucleotides upstream). (d) Average reactivity for the first, second, and third base of each coding sequence codon, and for the first, second, and third base of the $5^{\prime}$ UTR and 3' UTR, respectively, in the first and last 99 nucleotides of the coding region, last 48 nucleotides of the $5^{\prime}$ UTR, and first 48 nucleotides of the $3^{\prime}$ UTR. (e) Box-plot of base-normalized average CIRS-seq reactivities for protein-coding and non-coding RNAs, calculated on all transcript positions with sequencing depth $>50 \times$. 


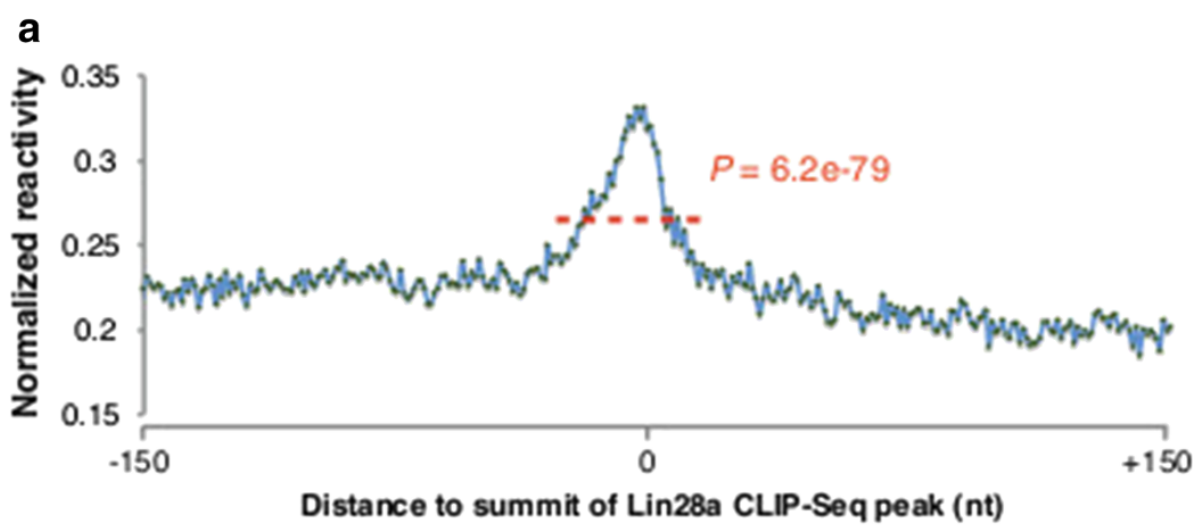

b

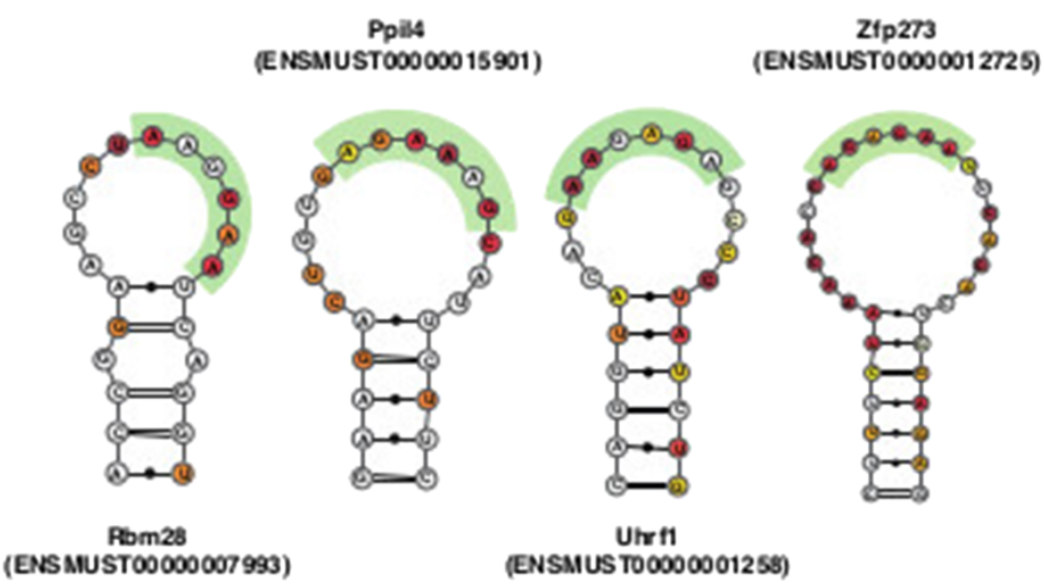

Figure 5 CIRS-seq reveals structural preferences of RNA binding proteins. (a) Average reactivity across 300 nucleotides surrounding summits of Lin28a peaks. (b) Representation of sample secondary structures for Lin28a binding sites. Bases are color coded according to their reactivity. The purine-rich motifs are highlighted in green.

structural ncRNAs. This is in agreement with the report that ncRNAs have higher melting temperatures than mRNAs, denoting higher structural stability [18].

Collectively, our data demonstrate that CIRS-seq can be used to obtain genome-wide information on native deproteinized RNA structures. Moreover, CIRS-seq methodology represents an important tool for the study of the structural binding specificities of RBPs.

\section{Conclusions}

We define for the first time the complete RNA structurome of mouse ESCs, by developing a high-throughput method for the analysis of RNA secondary structures in their native deproteinized conformation. This method achieved extremely high accuracy on validated secondary structures, and allowed the de novo prediction of RNA structures. Analysis of structural data for protein-coding RNAs revealed their strong structural partitioning between 5' UTRs, coding sequences, and 3' UTRs. Comparison with non-coding RNAs showed that ncRNAs are more structured than mRNAs, and that lincRNAs present an average structuring midway between protein coding and structural non-coding transcripts. We also reveal the structural requirements for binding of the RBP Lin28a, and demonstrate that our method can provide insight into the structural preferences of RBPs.

\section{Materials and methods}

\section{Cell culture}

Mouse E14 ESCs were grown on 0.1\% gelatin-coated plates and maintained in DMEM (4.5 g/L D-glucose) supplemented with $15 \%$ heat-inactivated fetal bovine serum, $0.1 \mathrm{mM}$ NEAA, $1 \mathrm{mM}$ sodium pyruvate, $0.1 \mathrm{mM} \mathrm{2-}$ mercaptoethanol, $25 \mathrm{U} / \mathrm{ml}$ penicillin, $25 \mu \mathrm{g} / \mathrm{ml}$ streptomycin and 1,500 U/ml LIF, as previously described [68].

\section{Quantitative RT-PCR}

Real-time quantitative PCR was performed using the SuperScript III Platinum One-Step Quantitative RT- PCR System (Invitrogen Carlsbad, CA, USA) as previously described [69]. The primers for the Rpph1 test transcript are provided in Table S1 in Additional file 1. 


\section{RNA-seq library preparation}

For RNA-seq library preparation, approximately $1 \mu \mathrm{g}$ of TRIzol (Invitrogen) isolated total RNA from ESCs was subjected to ribosomal RNA depletion using the Ribo-Zero Gold Kit (Epicentre Madison, Winsconsin, USA). rRNAdepleted RNA was used as the input for the RNA-seq library preparation using the TruSeq RNA Sample Prep Kit (Illumina) following the manufacturer's instructions.

\section{CIRS-seq}

Cell lysis and chemical probing, library preparation, and sequencing are detailed in the supplementary Materials and methods in Additional file 1.

\section{RNA quality assessment}

RNA sample quality was assessed with the Agilent Bioanalyzer 2100. All of the samples had an RNA integrity number ranging from 9.9 to 10 .

\section{Data analysis}

CIRS-seq data analysis, normalization and background subtraction, and transcript analysis are detailed in the supplementary Materials and methods in Additional file 1.

\section{Data access}

CIRS-seq and RNA-seq data have been deposited in the Gene Expression Omnibus (GEO) under accession number GSE54106. Additional datasets and the source code for the analysis tool are available at [70].

\section{Additional file}

Additional file 1: PDF file containing supplementary Materials and methods, Figures S1 to S5), Table S1, and supplementary references.

\section{Abbreviations}

CIRS: chemical inference of RNA structures; CMCT: N-cyclohexyl-N'-(2morpholinoethyl)carbodiimide metho-p-toluenesulfonate; DMS: dimethyl sulfate; ESC: embryonic stem cell; lincRNA: long intergenic non-coding RNA; IncRNA: long non-coding RNA; MFE: minimum free energy; ncRNA: non-coding RNA; PPV: positive predictive value; RBP: RNA binding protein; RT: reverse transcription; snRNA: small nuclear RNA; snoRNA: small nucleolar RNA; UTR: untranslated region.

\section{Competing interests}

The authors declare that they have no competing interests.

\section{Authors' contributions}

DI conceived the CIRS method. DI, FN and FA developed the library preparation. DI and FN developed computational methods and analyzed the data. SO supervised the experiments and wrote the manuscript. All authors read and approved the final manuscript.

\section{Acknowledgements}

This work was supported by the Associazione Italiana Ricerca sul Cancro (AIRC) IG 201111982.

\section{Author details}

${ }^{1}$ Human Genetics Foundation (HuGeF), via Nizza 52, Torino 10126, Italy. ${ }^{2}$ Dipartimento di Biotecnologie Chimica e Farmacia, Università di Siena, via Fiorentina, Siena 1-53100, Italy. ${ }^{3}$ Dipartimento di Scienze della Vita e Biologia dei Sistemi, Università di Torino, Via Accademia Albertina, Torino 13-10123, Italy.

Received: 29 July 2014 Accepted: 8 October 2014

Published online: 17 October 2014

\section{References}

1. ENCODE Project Consortium, Bernstein BE, Birney E, Dunham I, Green ED, Gunter C, Snyder M: An integrated encyclopedia of DNA elements in the human genome. Nature 2012, 489:57-74.

2. Djebali S, Davis CA, Merkel A, Dobin A, Lassmann T, Mortazavi A, Tanzer A, Lagarde J, Lin W, Schlesinger F, Xue C, Marinov GK, Khatun J, Williams BA, Zaleski C, Rozowsky J, Röder M, Kokocinski F, Abdelhamid RF, Alioto T, Antoshechkin I, Baer MT, Bar NS, Batut P, Bell K, Bell I, Chakrabortty S, Chen $X$, Chrast J, Curado J, et al: Landscape of transcription in human cells. Nature 2012, 489:101-108.

3. Derrien $T$, Johnson R, Bussotti G, Tanzer A, Djebali S, Tilgner H, Guernec G, Martin D, Merkel A, Knowles DG, Lagarde J, Veeravalli L, Ruan X, Ruan Y, Lassmann T, Carninci P, Brown JB, Lipovich L, Gonzalez JM, Thomas M, Davis CA, Shiekhattar R, Gingeras TR, Hubbard TJ, Notredame C, Harrow J, Guigó R: The GENCODE v7 catalog of human long noncoding RNAs: Analysis of their gene structure, evolution, and expression. Genome Res 2012, 22:1775-1789

4. Guttman M, Donaghey J, Carey BW, Garber M, Grenier JK, Munson G, Young G, Lucas AB, Ach R, Bruhn L, Yang X, Amit I, Meissner A, Regev A, Rinn JL, Root DE, Lander ES: lincRNAs act in the circuitry controlling pluripotency and differentiation. Nature 2011, 477:295-300.

5. Cabili MN, Trapnell C, Goff L, Koziol M, Tazon-Vega B, Regev A, Rinn JL: Integrative annotation of human large intergenic noncoding RNAs reveals global properties and specific subclasses. Genes Dev 2011, 25:1915-1927.

6. Bánfai B, Jia H, Khatun J, Wood E, Risk B, Gundling WE, Kundaje A, Gunawardena HP, Yu Y, Xie L, Krajewski K, Strahl BD, Chen X, Bickel P, Giddings MC, Brown JB, Lipovich L: Long noncoding RNAs are rarely translated in two human cell lines. Genome Res 2012, 22:1646-1657.

7. Guttman M, Russell P, Ingolia NT, Weissman JS, Lander ES: Ribosome profiling provides evidence that large noncoding RNAs do not encode proteins. Cell 2013, 154:240-251.

8. Tsai M-C, Manor O, Wan Y, Mosammaparast N, Wang JK, Lan F, Shi Y, Segal E, Chang HY: Long noncoding RNA as modular scaffold of histone modification complexes. Science 2010, 329:689-693.

9. Krummel DAP, Nagai K, Oubridge C: Structure of spliceosomal ribonucleoproteins. F1000 Biol Rep 2010, 2:39.

10. Nagai K, Muto Y, Pomeranz Krummel DA, Kambach C, Ignjatovic T, Walke S, Kuglstatter A: Structure and assembly of the spliceosomal snRNPs. Biochem Soc Trans 2001, 15-26.

11. Maenner S, Müller M, Fröhlich J, Langer D, Becker PB: ATP-Dependent roX RNA remodeling by the helicase maleless enables specific association of MSL proteins. Mol Cell 2013, 51:174-184.

12. Ilik IA, Quinn JJ, Georgiev P, Tavares-Cadete F, Maticzka D, Toscano S, Wan Y, Spitale RC, Luscombe N, Backofen R, Chang HY, Akhtar A: Tandem stem-loops in roX RNAs act together to mediate $X$ chromosome dosage compensation in Drosophila. Mol Cell 2013, 51:156-173.

13. Bertani S, Sauer S, Bolotin E, Sauer F: The noncoding RNA Mistral activates Hoxa6 and Hoxa7 expression and stem cell differentiation by recruiting MLL1 to chromatin. Mol Cell 2011, 43:1040-1046.

14. Kertesz M, Wan Y, Mazor E, Rinn JL, Nutter RC, Chang HY, Segal E: Genome-wide measurement of RNA secondary structure in yeast. Nature 2010, 467:103-107.

15. Underwood JG, Uzilov AV, Katzman S, Onodera CS, Mainzer JE, Mathews DH, Lowe TM, Salama SR, Haussler D: FragSeq: transcriptome-wide RNA structure probing using high-throughput sequencing. Nat Methods 2010 7:995-1001.

16. Lucks JB, Mortimer SA, Trapnell C, Luo S, Aviran S, Schroth GP, Pachter L Doudna JA, Arkin AP: Multiplexed RNA structure characterization with selective 2'-hydroxyl acylation analyzed by primer extension sequencing (SHAPE-Seq). Proc Natl Acad Sci U S A 2011, 108:11063-11068. 
17. Li F, Zheng Q, Ryvkin P, Dragomir I, Desai Y, Aiyer S, Valladares O, Yang J, Bambina S, Sabin LR, Murray JI, Lamitina T, Raj A, Cherry S, Wang L-S, Gregory BD: Global analysis of RNA secondary structure in two metazoans. Cell Rep 2012, 1:69-82.

18. Wan Y, Qu K, Ouyang Z, Kertesz M, Li J, Tibshirani R, Makino DL, Nutter RC, Segal E, Chang HY: Genome-wide measurement of RNA folding energies. Mol Cell 2012, 48:169-181.

19. Donahue CP, Fedor MJ: Kinetics of hairpin ribozyme cleavage in yeast. RNA 1997, 3:961-973.

20. Schroeder R, Grossberger R, Pichler A, Waldsich C: RNA folding in vivo. Curr Opin Struct Biol 2002, 12:296-300.

21. Donahue CP, Yadava RS, Nesbitt SM, Fedor MJ: The kinetic mechanism of the hairpin ribozyme in vivo: influence of RNA helix stability on intracellular cleavage kinetics. J Mol Biol 2000, 295:693-707.

22. Treiber DK, Williamson JR: Exposing the kinetic traps in RNA folding. Curr Opin Struct Biol 1999, 9:339-345.

23. Treiber DK, Williamson JR: Beyond kinetic traps in RNA folding. Curr Opin Struct Biol 2001, 11:309-314.

24. Shcherbakova I, Mitra S, Laederach A, Brenowitz M: Energy barriers, pathways, and dynamics during folding of large, multidomain RNAs. Curr Opin Chem Biol 2008, 12:655-666.

25. Pan T, Sosnick TR: Intermediates and kinetic traps in the folding of a large ribozyme revealed by circular dichroism and UV absorbance spectroscopies and catalytic activity. Nat Struct Mol Biol 1997, 4:931-938.

26. Antal M, Boros E, Solymosy F, Kiss T: Analysis of the structure of human telomerase RNA in vivo. Nucleic Acids Res 2002, 30:912-920.

27. Zemora G, Waldsich C: RNA folding in living cells. RNA Biol 2010, 7:634-641.

28. Pan T, Sosnick T: RNA folding during transcription. Annu Rev Biophys Biomol Struct 2006, 35:161-175.

29. Heilman-Miller SL, Woodson SA: Effect of transcription on folding of the Tetrahymena ribozyme. RNA 2003, 9:722-733.

30. Herschlag D: RNA chaperones and the RNA folding problem. J Biol Chem 1995, 270:20871-20874.

31. Russell R: RNA misfolding and the action of chaperones. Front Biosci 2008, 13:1.

32. Cristofari $G$, Darlix J-L: The ubiquitous nature of RNA chaperone proteins. Prog Nucleic Acid Res Mol Biol 2002, 72:223-268.

33. Weeks KM: Protein-facilitated RNA folding. Curr Opin Struct Biol 1997, 7:336-342.

34. Ding $Y$, Tang $Y$, Kwok CK, Zhang Y, Bevilacqua PC, Assmann SM: In vivo genome-wide profiling of RNA secondary structure reveals novel regulatory features. Nature 2014, 505:696-700.

35. Rouskin S, Zubradt M, Washietl S, Kellis M, Weissman JS: Genome-wide probing of RNA structure reveals active unfolding of mRNA structures in vivo. Nature 2014, 505:701-705.

36. Wells SE, Hughes JM, Igel AH, Ares M: Use of dimethyl sulfate to probe RNA structure in vivo. Methods Enzymol 2000, 318:479-493.

37. Tijerina P, Mohr S, Russell R: DMS footprinting of structured RNAs and RNA-protein complexes. Nat Protoc 2007, 2:2608-2623.

38. Metz DH, Brown GL: The investigation of nucleic acid secondary structure by means of chemical modification with a carbodiimide reagent. I. The reaction between $\mathrm{N}$-cyclohexyl-N'-beta-(4-methylmorpholinium) ethylcarbodiimide and model nucleotides. Biochemistry 1969, 8:2312-2328.

39. Metz $\mathrm{DH}$, Brown $\mathrm{GL}$ : The investigation of nucleic acid secondary structure by means of chemical modification with a carbodiimide reagent. II. The reaction between $\mathrm{N}$-cyclohexyl-N'-beta-(4-methylmorpholinium)ethylcarbodiimide and transfer ribonucleic acid. Biochemistry 1969, 8:2329-2342.

40. Ho NW, Gilham PT: Reaction of pseudouridine and inosine with $\mathrm{N}$-cyclohexyl-N'-beta-(4-methylmorpholinium)ethylcarbodiimide. Biochemistry 1971, 10:3651-3657.

41. Incarnato D, Krepelova A, Neri F: High-throughput single nucleotide variant discovery in E14 mouse embryonic stem cells provides a new reference genome assembly. Genomics 2014, 104:121-127.

42. Deigan KE, Li TW, Mathews DH, Weeks KM: Accurate SHAPE-directed RNA structure determination. Proc Natl Acad Sci U S A 2009, 106:97-102.

43. Hingerty $B$, Brown RS, Jack $A$ : Further refinement of the structure of yeast tRNAPhe. J Mol Biol 1978, 124:523-534.

44. Puglisi EV, Puglisi JD: Probing the conformation of human tRNA(3)(Lys) in solution by NMR. FEBS Lett 2007, 581:5307-5314.

45. Bayfield MA, Dahlberg AE, Schulmeister U, Dorner S, Barta A: A conformational change in the ribosomal peptidyl transferase center upon active/inactive transition. Proc Natl Acad Sci U S A 2001, 98:10096-10101.

46. Lyonnais S, Hounsou C, Teulade-Fichou M-P, Jeusset J, Le Cam E, Mirambeau G: G-quartets assembly within a G-rich DNA flap. A possible event at the center of the HIV-1 genome. Nucleic Acids Res 2002, 30:5276-5283.

47. Lambert NJ, Gu SG, Zahler AM: The conformation of microRNA seed regions in native microRNPs is prearranged for presentation to mRNA targets. Nucleic Acids Res 2011, 39:4827-4835.

48. Holmberg L, Melander $Y$, Nygård O: Probing the structure of mouse Ehrlich ascites cell 5.8S, $18 \mathrm{~S}$ and $28 \mathrm{~S}$ ribosomal RNA in situ. Nucleic Acids Res 1994, 22:1374-1382.

49. Alkemar G, Nygård O: Secondary structure of two regions in expansion segments ES3 and ES6 with the potential of forming a tertiary interaction in eukaryotic 40S ribosomal subunits. RNA 2004, 10:403-411.

50. Melander $Y$, Holmberg L, Nygård O: Structure of $18 \mathrm{~S}$ ribosomal RNA in native $40 \mathrm{~S}$ ribosomal subunits. J Biol Chem 1997, 272:3254-3258.

51. Ségault V, Will CL, Polycarpou-Schwarz M, Mattaj IW, Branlant C, Lührmann $\mathrm{R}$ : Conserved loop I of U5 small nuclear RNA is dispensable for both catalytic steps of pre-mRNA splicing in HeLa nuclear extracts. Mol Cell Biol 1999, 19:2782-2790.

52. Lukowiak AA, Granneman S, Mattox SA, Speckmann WA, Jones K, Pluk H, Venrooij WJ, Terns RM, Terns MP: Interaction of the U3-55 k protein with U3 snoRNA is mediated by the box $B / C$ motif of U3 and the WD repeats of U3-55 k. Nucleic Acids Res 2000, 28:3462-3471.

53. Granneman S, Pruijn GJM, Horstman W, van Venrooij WJ, Lührmann R, Watkins NJ: The hU3-55 K protein requires $15.5 \mathrm{~K}$ binding to the box $\mathrm{B} / \mathrm{C}$ motif as well as flanking RNA elements for its association with the U3 small nucleolar RNA in Vitro. J Biol Chem 2002, 277:48490-48500.

54. Yu E, Fabris D: Direct probing of RNA structures and RNA-protein interactions in the HIV-1 packaging signal by chemical modification and electrospray ionization fourier transform mass spectrometry. J Mol Biol 2003, 330:211-223.

55. Mougin A, Gottschalk A, Fabrizio P, Lührmann R, Branlant C: Direct probing of RNA structure and RNA-protein interactions in purified HeLa cell's and yeast spliceosomal U4/U6.U5 tri-snRNP particles. J Mol Biol 2002, 317:631-649.

56. Hajdin CE, Bellaousov S, Huggins W, Leonard CW, Mathews DH, Weeks KM: Accurate SHAPE-directed RNA secondary structure modeling, including pseudoknots. Proc Natl Acad Sci U S A 2013, 110:5498-5503.

57. Spitale RC, Crisalli P, Flynn RA, Torre EA, Kool ET, Chang HY: RNA SHAPE analysis in living cells. Nat Chem Biol 2013, 9:18-20.

58. Kwok CK, Ding Y, Tang Y, Assmann SM, Bevilacqua PC: Determination of in vivo RNA structure in low-abundance transcripts. Nat Commun 2013, 4:2971.

59. Reuter JS, Mathews DH: RNAstructure: software for RNA secondary structure prediction and analysis. BMC Bioinformatics 2010, 11:129.

60. Yu J, Vodyanik MA, Smuga-Otto K, Antosiewicz-Bourget J, Frane JL, Tian S, Nie J, Jonsdottir GA, Ruotti V, Stewart R, Slukvin II, Thomson JA: Induced pluripotent stem cell lines derived from human somatic cells. Science 2007, 318:1917-1920.

61. Cho J, Chang H, Kwon SC, Kim B, Kim Y, Choe J, Ha M, Kim YK, Kim VN: LIN28A is a suppressor of ER-associated translation in embryonic stem cells. Cell 2012, 151:765-777.

62. Shabalina SA, Ogurtsov AY, Spiridonov NA: A periodic pattern of mRNA secondary structure created by the genetic code. Nucleic Acids Res 2006, 34:2428-2437

63. Wan Y, Qu K, Zhang QC, Flynn RA, Manor O, Ouyang Z, Zhang J, Spitale RC, Snyder MP, Segal E, Chang HY: Landscape and variation of RNA secondary structure across the human transcriptome. Nature 2014, 505:706-709.

64. Gu S, Jin L, Zhang F, Sarnow P, Kay MA: Biological basis for restriction of microRNA targets to the $3^{\prime}$ untranslated region in mammalian mRNAs. Nat Struct Mol Biol 2009, 16:144-150.

65. Brown KM, Chu C-Y, Rana TM: Target accessibility dictates the potency of human RISC. Nat Struct Mol Biol 2005, 12:469-470.

66. Kertesz M, lovino N, Unnerstall U, Gaul U, Segal E: The role of site accessibility in microRNA target recognition. Nat Genet 2007, 39:1278-1284.

67. Clote P, Ferré F, Kranakis E, Krizanc D: Structural RNA has lower folding energy than random RNA of the same dinucleotide frequency. RNA 2005, 11:578-591

68. Neri F, Krepelova A, Incarnato D, Maldotti M, Parlato C, Galvagni F, Matarese F, Stunnenberg HG, Oliviero S: Dnmt3L antagonizes DNA methylation at 
bivalent promoters and favors DNA methylation at gene bodies in ESCs. Cell 2013, 155:121-134.

69. Galvagni F, Cartocci E, Oliviero S: The dystrophin promoter is negatively regulated by YY1 in undifferentiated muscle cells. J Biol Chem 1998, 273:33708-33713.

70. CIRS-seq: Chemical Inference of RNA Structure. [http://epigenetics. hugef-research.org/data/cirs.php]

doi:10.1186/s13059-014-0491-2

Cite this article as: Incarnato et al.: Genome-wide profiling of mouse

RNA secondary structures reveals key features of the mammalian transcriptome. Genome Biology 2014 15:491.

\section{Submit your next manuscript to BioMed Central and take full advantage of:}

- Convenient online submission

- Thorough peer review

- No space constraints or color figure charges

- Immediate publication on acceptance

- Inclusion in PubMed, CAS, Scopus and Google Scholar

- Research which is freely available for redistribution 